\title{
Editorial:
}

\section{Distributed filtering and control of complex networks and systems}

\author{
Guanrong $\mathrm{CHEN}^{1}$, Sergej ČELIKOVSKÝ ${ }^{2}$, Lei GUO ${ }^{3}$, Youmin $\mathrm{ZHANG}^{4}$, Tiancheng $\mathrm{LI}^{\ddagger 5}$ \\ ${ }^{I}$ Department of Electrical Engineering, City University of Hong Kong, Hong Kong, China \\ ${ }^{2}$ The Czech Academy of Sciences, Institute of Information Theory and Automation, Prague 18200, Czech Republic \\ ${ }^{3}$ School of Automation Science and Electrical Engineering, Beihang University, Beijing 100191, China \\ ${ }^{4}$ Department of Mechanical, Industrial and Aerospace Engineering, Concordia University, Montreal, Quebec H3G 1M8, Canada \\ ${ }^{5}$ MOE Key Laboratory of Information Fusion Technology, School of Automation, \\ Northwestern Polytechnical University, Xi'an 710072, China
}

E-mail: eegchen@cityu.edu.hk; celikovs@utia.cas.cz; lguo@buaa.edu.cn; ymzhang@encs.concordia.ca; t.c.li@nwpu.edu.cn

https://doi.org/10.1631/FITEE.2110000

In the last two decades, research on distributed filtering and control (FAC) has burgeoned into large-scale complex networks and systems. A distributed networked system, typically composed of a number of cost-effective dynamical nodes (agents) with limited sensing, computing, and communication capabilities, is gaining considerable popularity due to its low power consumption, simple installation, high performance, and strong reliability, as compared with the centralized setting. As a result, various networkinduced FAC systems, techniques, and algorithms have emerged, including node registration and control, network consensus and synchronization, multi-sensor data clustering/fusion, network topological design and analysis, and the like. In addition, it is important to have an insightful understanding of the way networks and systems behave and evolve from the FAC perspectives. This is often critical with respect to many important problems, such as managing a limited number of sensors for the best field-of-view coverage, tackling the intrinsic interactions and unknown correlations among dynamical nodes, trade-offs between performance and constraints, or achieving automation in noisy environments, just to name a few. These

\footnotetext{
${ }^{\ddagger}$ Correponding author

(c) Zhejiang University Press 2021
}

problems are imperative but also challenging for their multidisciplinary nature and inherent complexity, for which various theories, techniques, and algorithms are continuously being proposed and developed; however, more research effort and endeavors are needed.

This special issue is aimed at collecting highquality papers in the research field of distributed filtering and control of complex networks and systems; the final result includes two comprehensive reviews and nine original research papers.

The first paper by Da et al. (2021) is a survey of recent advances in multisensor multitarget tracking based on the random finite set (RFS) approach. While multisensor RFS fusion, which identifies an attractive yet challenging problem, has drawn much attention recently, this work serves as the first review in the literature using references that were published mainly in the last five years. Multisensor data fusion, which plays a fundamental role in distributed tracking, is briefly classified into two distinguishing groups in this review, data-level multitarget measurement fusion and estimate-level multitarget density fusion, which share and fuse local measurements and posterior densities between sensors, respectively. Important properties of each fusion rule, including optimality and sub-optimality, are detailed therein. In 
particular, two robust multitarget density averaging approaches, i.e., arithmetic- and geometric-average fusion, are addressed with regard to various posterior distributions. Meanwhile, research trends and remaining challenges are highlighted.

The second paper by Wen et al. (2021) reviews recent progress on distributed economic dispatch in smart grids. Achieving an efficient distributed economic dispatch (DED) strategy for the smart grid (SG) in the presence of multiple generators plays a paramount role in obtaining various benefits of such new-generation power systems, such as easy implementation, low maintenance cost, high energy efficiency, and robustness. This paper focuses in particular on the pertinent literature published since 2015, which is categorized into distributed discrete-time and continuous-time economic dispatches of the SG in the presence of multiple generators. In addition, some future research directions in DED for the SG are discussed.

The third paper by Yang et al. (2021) addresses the economic dispatch problem of the smart grid under vicious denial of service (DoS) attack. Taking the actual situation of power generation as a starting point, a new distributed optimization model is established, which takes the environmental pollution penalty into account. To save the bandwidth, a novel distributed event-triggered scheme is proposed to keep the resilience and economy of a class of cyber-power systems when the communication network is subject to a malicious DoS attack. An improved multi-agent consensus protocol based on the gradient descent idea is subsequently designed to solve the minimization problem, and the prerequisites to minimize the system power generation cost are analyzed from the perspectives of optimality and stability.

The fourth paper by Wang et al. (2021) presents a new event-triggered Kalman consensus filter (ET$\mathrm{KCF}$ ). The proposed algorithm is based on information freshness, which is calculated as the age of information (AoI) of the sampled data. It integrates the event-triggered mechanism, updated information method, and KCF algorithm to estimate the concentration of pollutants in the aircraft more efficiently. The disclosed method also takes into account the influence of data packet loss and the loss of aircraft communication path, and presents an AoI-freshnessbased threshold selection method for ET-KCF, which compares the packet AoI to the minimum average AoI of the system. This approach can clearly reduce energy consumption, as the transmission of expired information is reduced. The convergence of the algorithm is proved. Simulation results show that the algorithm has better fault tolerance compared to the existing KCF and features lower power consumption than other ET-KCFs.

The fifth paper by Torres et al. (2021) introduces an asymptotic observer of constant gain for nonlinear systems that have linear input. This allows the observer design to be formulated within the linear matrix inequality paradigm, provided that a strictly positive real condition between the input disturbance and the output is fulfilled. The proposed observer is then applied to a large class of nonlinear chemostat dynamical systems. Under some typical assumptions, the necessary change of chemostat state coordinates exists, allowing the use of the constant-gain observer. The developed theory is tested by estimating pollutant concentration in a Spirulina maxima wastewater treatment facility.

The sixth paper by Wu et al. (2021) extends traditional (single-target) hybrid systems to multitarget hybrid systems by focusing on the multimaneuvering target tracking system. This system consists of a continuous, discrete, and switchable state, as well as a discrete time-constant state; based on these, the paper presents an exact derivation of a solution for the multi-model generalized labeled multi-Bernoulli filter.

The seventh paper by Wang (2021) analyzes the convergence of time-varying networks, and applies the results for the cooperative control of nonlinear multiagent systems (MASs) with unknown control directions (UCDs). It is proven therein that, if the time-varying networks are cut-balanced, the convergence of nonlinear MASs with non-identical UCDs is achieved using the presented algorithms. A critical feature of this application is that the designed algorithms can be used to deal with non-identical UCDs by employing conventional Nussbaum-type functions.

The eighth paper by Rehák et al. (2021) proposes an algorithm for a leader-follower synchronization of an MAS composed of linear agents with time delays. The presence of different delays in various agents can cause a synchronization error that does not converge to zero. However, the norm of this error can be 
bounded, as demonstrated in the paper. The proof of the main results is formulated by means of linear matrix inequalities, and the size of the problem is not dependent on the number of agents. Results are illustrated through examples, highlighting the fact that the steady-state error is caused by heterogeneous delays. The paper finally demonstrates the capacity of the proposed algorithm to achieve synchronization with up to a permissible small error.

The ninth paper by Sun et al. (2021) studies the containment control problem for high-order heterogeneous nonlinear MASs under distributed eventtriggered schemes. For containment control with little communication among the agents, a distributed event-triggered control scheme is proposed based on the backstepping technique, Lyapunov function, and neural networks. Then, the result is extended to the self-triggered control setting to avoid continuous monitoring of state errors. The developed protocols and rules ensure the convergence of the output for each follower to the convex hull spanned by multiple leader signals within certain error bounds. Moreover, it is achieved that no agent exhibits Zeno behavior.

The tenth paper by Hu et al. (2021) studies the state consensus of an MAS, where new agent groups are added dynamically into the original MAS. The paper analyzes the feasibility of dynamically adding agent groups under different common forms of network topologies, and obtains four feasible schemes theoretically, including one scheme that is the best in actual industrial production. Subsequently, the paper carries out a dynamic modeling of MAS using the scheme determined as the best. Impulsive control theory and Lyapunov stability theory are used to analyze the conditions under which the whole MAS with dynamic join characteristics achieves state consensus.

The last paper by Yang et al. (2021) examines the finite-time formation control of MASs with region constraints, where multiple agents have first-order dynamics with a common target area. A novel control algorithm is proposed using local information and through interactions. If the communication graph is undirected and connected, and the desired framework is rigid, it is then proved that the controller can be used to solve the formation problem within the target area; that is, all agents can enter the desired region in finite time while reaching and maintaining the desired formation shapes.
The guest editors would like to thank all the authors for their fine contributions to this special issue, and appreciate the dedication from all the reviewers for their time and effort. Great support of the editorial office throughout the entire process also deserves our appreciation. It is our hope that the selected papers indeed capture some latest major scientific developments and can serve as a springboard for further research progress.

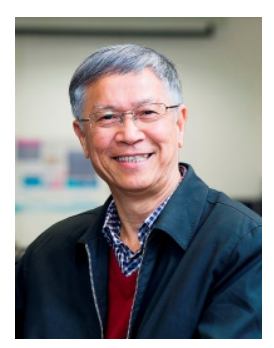

Prof. Guanrong CHEN received his MS degree in computer science from Sun Yat-sen University, China, in 1981, and his $\mathrm{PhD}$ degree in applied mathematics from Texas A\&M University, USA, in 1987. He has been a chair professor and the founding director of the Centre for Chaos and Complex Networks at City University of Hong Kong, China since 2000; before that, he was a tenured professor at the University of Houston, USA. Prof. CHEN received the State Natural Science Award of China in 2008, 2012, and 2016. He was elected as an IEEE fellow in 1997, and is now a life fellow. He was awarded the 2011 Euler Gold Medal, Russia and conferred honorary doctorates by the Saint Petersburg State University, Russia, in 2011 and by the University of Le Havre, France, in 2014. He is a member of the Academy of Europe and a fellow of the World Academy of Sciences. He has been a highly cited researcher in engineering since 2009 according to Thomson Reuters/Clarivate. Currently, he is the Editor-in-Chief of Int $J$ Bifurc Chaos. His main research pursuit is in nonlinear system control and dynamics, as well as complex networks.

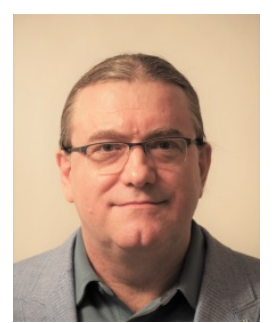

Prof. Sergej ČELIKOVSKÝ received his MS degree in applied mathematics from the Optimal Control Department, Moscow State University, in 1984, and his $\mathrm{PhD}$ degree in Technical Cybernetics from the Czechoslovak Academy of Sciences, Czech Republic, in 1988. He was a visiting researcher at University of Twente, the Netherlands, in 1996, at Department of Mechanical and Automation Engineering of Chinese University of Hong Kong, China, in 1998, and at CINVESTAV del I.P.N. in Mexico during 1998-2000. Currently, he is a research fellow and head of the Department of Control Theory in the Institute of Information Theory and Automation, Czech Academy of Sciences and a professor at the Department of Control Engineering, Czech Technical University in Prague. He has been an associate member of the Centre for Chaos and Complex Networks at City University of Hong Kong, China since 2003. Prof. ČELIKOVSKÝ has been a senior member of the IEEE since 2003, and he was a member of general assembly of the 
European Union Control Association during 2011-2017. He received the SICE 2011 Award for Outstanding Paper from the Society of Instrument and Control Engineers, Japan, and was awarded EEA (French Association of Professors and Researchers in Electrical and Information Sciences) Demonstrator Paper Prize at the IFAC World Congress 2017 in Toulouse. He authored or co-authored over 70 journals papers, receiving over 2000 citations according to Web of Science, and published a monograph at Prentice Hall. He has been a member of the editorial board or international program committee of various recognized journals and conferences, among them IEEE Trans Autom Contr during 2006-2010 and IFAC World Congress in 2014 and 2017. He served as a subarea chair of IFAC NOLCOS in 2007 and 2010. Currently, he is a subject editor of J Franklin Inst and an associate editor of Int J Bifurc Chaos. His current research interests include nonlinear systems, mechanical systems and robotics, complex systems and networks, chaotic systems, optimal control and optimization, robust control, stabilization and estimation, and modeling and control of biosystems.

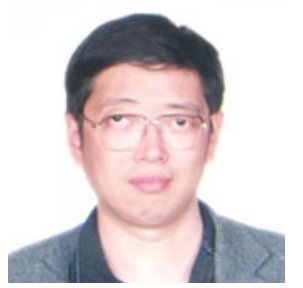

Prof. Lei GUO received his BS degree in fundamental mathematics and his MS degree in operational research and cybernetics from Qufu Normal University, China, in 1988 and 1991, respectively, and his $\mathrm{PhD}$ degree in control engineering from Southeast University, China, in 1997. From 1999 to 2000, he was a research fellow with the IRCCyN, France. From 2000 to 2003, he was a research fellow at University of Glasgow, Loughborough University, and University of Manchester Institute of Science and Technology, all in UK. Currently, he is a professor with the School of Automation Science and Electronic Engineering, Beihang University, China. Prof. GUO is an awardee of the National Science Fund for Distinguished Young Scholars of China and a Changjiang Distinguished Professor of the Ministry of Education of China. He has published more than 170 papers, and served as an editor for several journals. His research interests include robust control, stochastic systems, fault detection, filter design, and nonlinear control with their applications in aerospace systems.

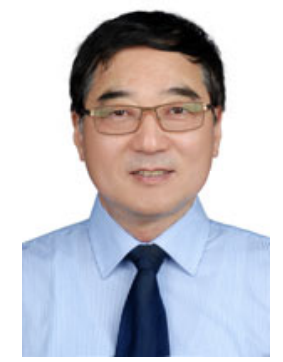

Prof. Youmin ZHANG received his BS, $\mathrm{MS}$, and $\mathrm{PhD}$ degrees in automatic control from Northwestern Polytechnical University, China, in 1983, 1986, and 1995 , respectively. He is currently a professor with the Department of Mechanical, Industrial and Aerospace Engineering and the Concordia Institute of Aerospace Design and Innovation, Concordia University, Canada. Prof. ZHANG is a fellow of the Canadian Society for Mechanical Engineering (CSME), a senior member of AIAA and IEEE, and a member of the technical committee for several scientific societies, including currently being president of the International Society of Intelligent Unmanned Systems (ISIUS). He has authored five books and published more than 500 journal and conference papers. He is an editorial board member, editor/associate editor of several international journals. He has been general or program chair several times for several unmanned systems and renewable energies relevant international conferences. His current research interests include fault detection and diagnosis (FDD) and fault-tolerant control (FTC), guidance, navigation, and control (GNC), and signal/image processing techniques with applications in unmanned aerial/space/ground/surface vehicles, smart grids, and smart cities.

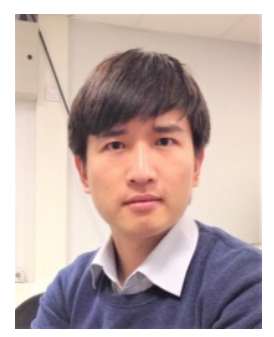

Prof. Tiancheng LI received two BS degrees from Harbin Engineering University, China, in 2008, and two $\mathrm{PhD}$ degrees, the first from London South Bank University, UK, in 2013 and the second from Northwestern Polytechnical University (NPU), China, in 2015. $\mathrm{He}$ is currently a professor with the School of Automation, NPU. Prior to this, he was a postdoctoral researcher with the BISITE Group, University of Salamanca, Spain, from June 2014 to the fall of 2018, and a visiting scholar with the Vienna University of Technology, Austria in the summer of 2017 and in the fall of 2018. He is an associate editor of Front Inform Technol Electron Eng and Adv Distrib Comput Artif Intell J. His research focuses on distributed information fusion, and data-driven algorithms for target detection, tracking, and forecasting. 